\title{
La retorica nella narrativa del Boccaccio. L'“Elegia di Madonna Fiammetta"
}

Renato Barilli

L'impianto retorico del Decamerone è dichiarato dal Boccaccio nel Proemio dell'opera, dove egli individua sia la materia, sia la categoria di soggetti cui ritiene opportuno dedicare le sue attenzioni. La materia è quella delle pene d'amore, in cui si considera particolarmente esperto. Egli confessa infatti di essere appena uscito da un'intensa avventura sentimentale, e dunque di poter trasmettere ad altri il frutto che ne ha ricavato. Sono rosí presenti i tre fini classici di ogni discorso retorico: il docere, il movere, il delectare. Il docere, poiché si tratta di comunicare taluni ammaestramenti sul decorso delle passioni; il movere, perché appunto il discorso verte su di queste, cioè su una materia viva di emozioni; e inoltre data la loro natura mondana, il trattarle non puó mancare di raggiungere un effetto dilettoso e piacevole.

Ma è assai piú interessante considerare a quale categoria di persone l'autore intenda indirizzare il suo particolare discorso retorico. Sappiamo bene che la retorica interviene là dove si abbia a che fare con un pubblico popolare, di uomini della strada, non particolarmente dotti ed esperti in qualche materia, ma forti solo della normale dotazione appartenente al senso comune. Ebbene, dove trovare una categoria piú rispondente a simili caratteristiche di quella delle donne? Esse infatti sono state le vittime di una millenaria discriminazione che le ha escluse dall'educazione e dalla vita pubblica, dalla conduzione degli affari. Un simile stato di fortuna ha appena cominciato a mutare al giorno d'oggi, immaginiamoci quindi quanto esso dovesse pesare in una società pur relativamente evoluta come I'Italia del Trecento. Anche se, beninteso, le donne che Boccaccio frequenta e a cui si rivolge sono di alta condizione, la migliore dell'epoca: di provenienza aristocratica, o uscite da una ricca borghesia che proprio in quel momento stava 
sorgendo. Ma ciò non toglie che esse vivessero "ristrette da' voleri, da' piaceri, da' comandamenti de' padri, delle madri, de' fratelli e de' mariti," (p. 4). ${ }^{1}$ Inoltre alle donne di quello strato elevato non era concesso il modo pur faticoso e ingrato di passare il tempo attendendo ai lavori domestici che invece si imponeva alle donne del popolo. Esse si trovavano pertanto "nel piccolo circuito delle loro camere racchiuse . . . e quasi oziose sedendosi, volendo e non volendo in una medesima ora." Laddove agli uomini di quella medesima condizione sociale non mancava ". . I'andare attorno, udire e vedere molte cose, uccellare, cacciare, pescare, cavalcare, giucare e mercatare" (p. 5). Vale a dire, essi erano chiamati a frequentare il foro, che è anche il luogo deputato per esercitare la retorica, ben diverso a sua volta dalle aule accademiche dove invece pochi dotti e filosofi si possono dare al difficile e arido dibattito dialettico.

A quel punto il Boccaccio veniva a trovarsi di fronte a una scelta di base: o equiparare la camera delle donne al foro, oppure andare fino in fondo nella logica della camera, cioè di un luogo separato, chiuso e silenzioso: dichiarare pertanto la fine del discorso retorico, cioè dell'uso pubblico del discorso verbale, pronunciato ad alta voce e rivolto a interlocutori presenti in carne e ossa, darsi invece a procedimenti intuitivi di interpretazione della vita emotiva delle donne, per offrirne poi un resoconto ai lettori attraverso una descrizione analitica. Questo anche in forza del ragionamento, dettato dal criterio della verosimiglianza, secondo cui le donne, appunto perché di scarsa istruzione, non erano in grado di esprimersi con eloquenza, e quindi toccava all'autore decifrare il loro inevitabile silenzio. Si aggiungeva anche un'altra considerazione, questa volta di ordine psicologico: la passione amorosa ci sconvolge, ci toglie la parola, e quindi favorisce anch'essa il risultato del silenzio.

Questo esito, della passione muta che toglie la possibilità della parola invocando la mediazione descrittiva del narratore, sarà imboccato dall'età "moderna," se prendiamo questo termine nella sua accezione accademica e lo consideriamo quindi coestensivo dei secoli che vanno dalla fine del ' 400 a tutto l'Ottocento. È anche l'arco di tempo, ci direbbero Marshall McLuhan e Walter Ong, ${ }^{2}$ in cui la tipografia favorisce appunto i procedimenti silenziosi della lettura effettuati nel segreto delle proprie stanze, provocando la totale disgrazia del discorso pronunciato in pubblico su cui si fondava la retorica. In ritardo di circa un secolo rispetto all'invenzione di Gutenberg, il grande commentatore aristotelico Ludovico 
Castelvetro accuserà appunto il Boccaccio di far parlare troppo una delle eroine del Decamerone, Ghismonda, cui il padre, il principe Tancredi, fa uccidere l'amante di basso livello sociale. ${ }^{3}$ Le argomentazioni del Castelvetro si appoggeranno precisamente sui due cardini della verosimiglianza, cioè sul fatto che Ghismonda, malgrado i suoi nobili natali, non poteva disporre di un'eloquenza cosí ampia e forbita; e che inoltre, essendo choccata dall'uccisione tragica dell'amante, non poteva trovarsi nella possibilità di parlare. Un buon erede delle preoccupazioni del Castelvetro, agli inizi dell'Ottocento, sarà il Manzoni, che nel pronunciare il celebre "Addio ai monti" sentirà di doversi sostituire a Lucia, cui per le medesime ragioni è possibile accreditare soltanto un complesso di sensazioni confuse, e soprattutto ineffabili. ${ }^{4}$

Ma alcuni secoli prima il Boccaccio aveva optato per la soluzione opposta: far sí che la camera si aprisse a un tratto alle dimensioni sonore e pubbliche del foro, divenendo in un certo senso il foro della vita passionale. Per ottenere un simile risultato, occorreva appunto che un uomo istruito di lettere e di retorica come l'autore imprestasse quelle sue doti alle povere colleghe in pene d'amore, altrimenti sprovvedute e indifese. $\mathrm{O}$ in altre parole, il Boccaccio ha preceduto Flaubert nel pronunciare il fatidico: "Madonna Ghismonda c'est moi." In un certo senso, egli è andato anche piú a fondo nell'equiparazione, dato che Flaubert, continuatore del Manzoni sotto questo aspetto, si limita a ricostruire i "pensieri" di Madame Bovary in forma indiretta, mentre il Boccaccio impresta letteralmente la sua eloquenza alle eroine del Decamerone. Il fatto è che egli vive e opera in un'età pre-moderna, pre-gutenberghiana, dove è inconcepibile darsi alle virtú analitiche della lettura. Lo strumento principale della comunicazione è appunto il discorso retorico, esercitato in tutta la sua opulenza, avendo come obiettivo finale la declamazione pubblica, rispetto alla quale la scrittura costituisce appena una fase transitoria. Il circuito bocca-orecchi mantiene ancora tutta la sua autorevolezza, non minacciata dalle virtú dell'occhio e della lettura. Ciò spiega anche la ricchezza del periodare boccaccesco, e la sua relativa sfortuna, quando poi subentreranno i tempi e le esigenze economiche dell'età "moderna," affidate all'occhio e alla stampa. D'altra parte, non dimentichiamo che noi viviamo in un'epoca postmoderna in cui le potenzialità positive della retorica sono state ampiamente rivalutate. ${ }^{5}$ I narratori contemporanei hanno sollevato i personaggi dagli stretti obblighi della verosimiglianza psicologica, hanno restituito loro il diritto di dibattere, di parlare, di scambiare le parti con l'autore. 
Basti pensare per tutti al caso di Pirandello, che sotto molti aspetti può essere considerato un corrispondente a distanza del Boccaccio, come lui impegnato a prestare la sua abilità avvocatesca a certi "umiliati e offesi" altrimenti incapaci di esprimersi. ${ }^{6}$

Se accettiamo di scorgere nel Boccaccio, essenzialmente, un logografo, un avvocato al servizio delle donne, onde difendere la loro vita sentimentale, ne viene un risultato a sorpresa. Il piú bell'esempio di una simile attività retorica non lo troveremo in alcuna delle cento novelle del Decamerone, bensí in un'opera di datazione incerta, ma sicuramente precedente di qualche anno, l'Elegia di Madonna Fiammetta, che si può considerare come una delle novelle del capolavoro ma premiata da uno sviluppo quantitativo eccezionale, per cui essa supera di parecche misure il piú lungo dei racconti narrati nelle due giornate. $\mathrm{E}$ in questi casi la quantità è anche qualità, la lunghezza consente una piú ricca strumentazione al discorso retorico.

In genere, i critici hanno riconosciuto il grande valore dell'Elegia di Madonna Fiammetta, ma tendendo però a farne un caso autonomo, diverso e forse meno maturo rispetto al Decamerone, legato a una fase piú astrattamente umanistica del Boccaccio. Ma una valutazione di questo genere è stata forse influenzata da alcuni pregiudizi di carattere "moderno": come se i meriti della narrativa boccaccesca si dovessero valutare sul metro della verosimiglianza e del realismo, secondo l'immagine arbitraria di un Boccaccio che anticipa appunto la grande narrativa moderna culminante nell'Ottocento, nella capacità di prestare attenzione ai casi degli umili, di avvalersi dello stile basso, di abbracciare condizioni di vita e di fortuna assai diverse tra loro. Un Boccaccio maestro di psicologia "silenziosa" e quindi di virtú descrittive, di prosa funzionale e analitica. Virtú, tutte queste, al cui confronto stridono inevitabilmente le doti retoriche ed eloquenti che invece traspaiono nei discorsi delle donne innamorate del Decamerone, e che già avevano toccato un vertice di magnificenza nel caso di Madonna Fiammetta. Ma se i ragionamenti d'amore del Decamerone, lungi dall'essere delle concessioni fatte dall'autore a un gusto letterario antico o medievale, ne sono in realtà il fulcro, il motivo ispiratore centrale, viene da ciò la necessità di rivalutare l'Elegia, di giudicarla anzi come una specie di prova generale delle analoghe prestazioni poi ricorrenti nel Decamerone, o comunque di considerarla in tutto e per tutto omogenea ad esse, e anzi superiore, per quel grado di maggiore sviluppo almeno quantitativo cui si accennava. 
In quest'opera l'autore non riserva a se stesso alcun "cantuccio," neppure ricorrendo allo stratagemma del proemio. La donna ci parla dal principio alla fine, e anche per questo verso il risultato è piú radicale, l'equiparazione "Madonna Fiammetta c'est moi" ancora piú spinta. D'altronde, un qualche tributo alla verosimiglianza doveva pur essere pagato. Nel Decamerone, appunto nel Proemio, l'autore si incarica di precisare che intende assumere la funzione di logografo a favore delle donne. Qui, non potendo egli uscire in una simile confessione, tocca a Fiammetta di proporre una giustificazione tutto sommato meno convincente: sarebbe Amore a trasmetterle la necessaria eloquenza di cui diversamente essa non potrebbe dare prova con le sole sue forze.

In realtà, già in questa "prova generale" è il Boccaccio che impresta alla giovane una serie inesauribile di orazioni, concioni, declamazioni, indirizzate a tutte le dramatis personae della vicenda, presenti e assenti, vicine e lontane, umane e divine. Il luogo della storia è dato dalle camere private in cui questa nobildonna napoletana vive la sua travolgente passione per un giovane straniero, Panfilo (il nome dell'amante, come anche il suo proprio, sono dichiaratamente di convenienza). Solo raramente si esce da quelle stanze per frequentare luoghi pubblici. Eppure, come già si notava, Fiammetta respinge sdegnosamente lo statuto del silenzio, e anzi pretende di riempire quello spazio limitato con i suoi ragionamenti ad alta voce. E del resto conviene ricordare che essa assume già in partenza la forma interlocutoria epistolare, rivolgendosi a noi ipocriti lettori, o meglio discriminando tra il pubblico maschile e quello femminile (il primo è considerato insensibile e prevenuto). Entro il "voi" del discorso di base, indirizzato a tutte le donne del mondo, si aprono poi le virgolette ideali di tante altre allocuzioni interne, attraverso le quali Fiammetta si rivolge a se stessa, al marito, alla nutrice, a Panfilo, alle donne con cui l'amante la tradisce, alla Fortuna, alle divinità, all'Amore, e cosí via. Alcuni di questi interlocutori intervengono a loro volta, e cosí alimentano il grande circuito orale-aurale. Fatto sta che i brani descrittivi-narrativi sono quantitativamente minori rispetto a quelli delle orazioni in forma diretta; del resto anch'essi si devono pur sempre considerare preceduti dalle virgolette dell'allocuzione di base, della straripante epistola che Fiammetta rivolge urbi et orbi.

Tra i vari artifici forniti dalla tradizione retorica cui il Boccaccio attinge a piene mani, ve n'è uno che caratterizza un intero capitolo dell'operetta, l'ottavo e penultimo, situato prima del "con- 
gedo" ufficiale. Si tratta dell'espediente classico dell'elenco di esempi, dell'evocazione di una serie di casi illustri, di vicende simili a quelle della protagonista, patite da personaggi consacrati dal mito, dalla storia, dalla letteratura. Nel tracciare questa galleria di esempi il Boccaccio rivela la sua conoscenza fin troppo ampia della letteratura classica, tra Ovidio e Virgilio e Seneca, con qualche apertura anche alla materia romanza, per cui nella sfilata compaiono pure Tristano e Isotta, accanto a Piramo e Tisbe, a Didone, Fedra, Cleopatra ecc. Parrebbe questa la parte piú stucchevole dell'operetta, da mettere per intero nel conto di una soggezione retorica non riscattata, ovvero di quell'eccesso di fede nelle humanae litterae che aduggerebbe alquanto l'Elegia, secondo il parere di molti critici. E beninteso qualche stonatura in tal senso è da riconoscere. D'altronde, per carità, non si tenti di giocare una carta di recupero improntata a un gusto "moderno." Non si pretenda di scoprire, cioè, nel ritratto che Fiammetta fa di sé confrontandosi con le sue tristi colleghe dell'antichità, pregi di realismo moderno, quali sarebbero la presenza di qualche tratto ambientale capace di renderci il sapore della Napoli trecentesca; oppure qualche finezza introspettiva degna appunto della psicologia analitica di taglio moderno. Nulla di tutto ciò. I fondali in cui Fiammetta recita la sua passione sono stereotipati e di maniera, convertibili a piacere con quelli di altre vicende amorose di altri tempi e luoghi; e inoltre, dovrebbe ormai esser chiaro, i suoi gusti concedono ben poco al gusto per l'interiorità e il "sottovoce" che invece è cosí caro alla sensibilità "moderna," da Manzoni a Flaubert.

Dove allora rintracciare la diversità di Fiammetta rispetto a tutte le infelici vittime delle pene d'amore consacrate dal mito e dalla leggenda? La nostra protagonista si rivela consapevole di una differenza sostanziale tra il caso suo e quello di costoro, differenza che per un verso ci riporta alla natura retorica dell'approccio boccaccesco, ma per altro verso è anche alle origini di alcune proprietà strutturali del romanzo moderno. Fiammetta chiarisce molto bene che le storie sentimentali delle celebri donne da lei evocate erano per lo piú votate a una fine tragica, destinate a una catastrofe, il che le rende incommensurabili alla sua propria storia per l'altezza delle loro sofferenze. Ma nello stesso tempo quelle sofferenze erano anche caratterizzate dalla brevità della loro durata, e proprio perché appariva ineluttabile il sacrificio ultimo. Invece l'amore di Fiammetta è di natura media; essa sa bene che come lei tante altre donne ci sono passate e ci passeranno; e anche se non lo vuole ammettere del tutto, aderisce in sostanza alla 
saggezza della balia, che le ricorda come i grandi amori si dimentichino, e possano essere sostituiti da altri. E poi, chissà, Panfilo un giorno potrebbe ritornare. $\mathrm{O}$ in altre parole, i casi illustri da lei ricordati terminano in genere con la morte delle protagoniste, invece la sua esperienza personale si muove nel solco dell'aureo detto "fiñché c'è vita c'è speranza." È insomma una vicenda aliena dal tragico, votata invece a quel dramma di carattere quotidiano, a quel misto di gioie e di sofferenze di cui è impastata ogni vita comune, e che in effetti costituirà uno degli ingredienti del romanzesco "moderno."

Inoltre, riconosciuto il carattere normale, mediocre, potremmo anche dire "borghese" di questa educazione sentimentale cui ognuno di noi è votato, risulta anche quanto sia pertinente nei suoi confronti il discorso retorico, con le tre doti di fondo che esso comporta, il movere, il docere, il delectare. Proprio perché si continua a vivere, è utile ricevere ammaestramenti da chi è già passato per certi travagli, oppure renderci autori a nostra volta di un simile servizio a chi è desideroso di apprendere, e di imparare cosí a dominare una materia sentimentale-emotiva, la cui esposizione non va mai disgiunta, oltretutto, da un certo grado di diletto. Le donne, in particolare, hanno in tutto ciò un ruolo fondamentale e prioritario, perché di sicuro "sentono" di piú degli uomini, sono immerse fino al collo nella vita emotiva, mentre per altro verso si trovano costrette a una condizione mediana, aliena da eroismi. È quanto Fiammetta fa risultare molto bene confrontandosi addirittura con Ulisse. L'eroe di Itaca ha dovuto tollerare tante traversie, eppure è meno degno di compassione rispetto a lei stessa, dato che, essendo un maschio, è stato allenato fin da piccolo alla sofferenza; inoltre, per la sua condizione sociale di condottiero, sapeva bene già in partenza di dover rischiare per conseguire la gloria. Invece le donne, nelle battaglie dell'amore extraconiugale, non hanno per premio la gloria, se l'esito è positivo, mentre ne hanno vergogna, disonore, se la relazione è infelice e traspare alla luce del sole. Per queste ragioni la loro educazione sentimentale è assai importante, detiene un posto centrale nella condizione umana, e dunque bene ha fatto il Boccaccio ad assumere il compito storico di darle gli opportuni strumenti retorici che le consentissero di manifestarsi in pubblico, di parlare a tutti. 


\section{NOTE}

1 Le citazioni del Decamerone fanno riferimento all'edizione a cura di E. Bianchi (Milano-Napoli: Ricciardi, 1952). Quelle all'Elegia di Madonna Fiammetta all'ed. a cura di C. Salinari e N. Sapegno, ivi.

2 Per il primo, rimando all'ormai classico The Gutenberg Galaxy, the Making of Typographical Man (London: Routledge Kegan Paul, 1962); per il secondo, ha particolare rilevanza nel presente discorso The Presence of the Word (New Haven: Yale University Press, 1967, trad. it. Bologna: Mulino, 1970 con intro. mia).

3 Cfr. il mio studio "Semiologia e retorica nella lettura del Decameron." Il Verri, 35-6 (1971), 27-48; quanto al Castelvetro, rinvio allo studio che gli dedico in Poetica e retorica, 2nd ed. (Milano: Mursia, 1984).

4 Infatti, al termine del brano, l'A. precisa di aver reso il "genere," ma non la forma delle riflessioni di Lucia: "Di tal genere, se non tali appunto, erano i pensieri di Lucia. ...."

5 Rimando, oltre a Poetica e retorica, cit., all'excursus globale attraverso le fortune e sfortune dell'ars dicendi che ho svolto in Retorica, 2nd ed. (Milano: Isedi, 1983).

6 Cfr. ancora le considerazioni svolte nel saggio cit. Semiologia e retorica ecc., poi svolte in La linea Svevo-Pirandello, 3rd ed. (Milano: Mursia, 1981). 